\title{
Are probiotics effective in management of irritable bowel syndrome?
}

Asieh Hosseini ${ }^{1}$, Shekoufeh Nikfar ${ }^{2,3}$, Mohammad Abdollahi ${ }^{4}$

1Razi Institute for Drug Research, Tehran University of Medical Sciences, Tehran, Iran
2Department of Pharmacoeconomics and Pharmaceutical Administration, Faculty
of Pharmacy, Tehran University of Medical Sciences, Iran
${ }^{3}$ Food and Drug Laboratory Research Center, Food and Drug Organization, Ministry
of Health and Medical Education, Iran
4Faculty of Pharmacy and Pharmaceutical Sciences Research Center, Tehran University
of Medical Sciences, Tehran, Iran

Submitted: 17 April 2012

Accepted: 23 April 2012

Arch Med Sci 2012; 8, 3: 403-405

DOI: $10.5114 /$ aoms.2012.29396

Copyright (c) 2012 Termedia \& Banach

Irritable bowel syndrome (IBS) is a chronic disorder of the gut that is common in the world, causing a financial burden to health service and people. IBS is generally divided into several types including diarrhea-predominant IBS (IBS-D), constipation-predominant IBS (IBS-C), and mixed IBS (sometimes constipation, sometimes diarrhea). Despite the high prevalence of IBS, the exact pathophysiology of IBS is not clear but autonomous nervous system dysfunction seems the most important factor [1]. The management of IBS is often based on severity of disease, and the patient or practitioner preferences. Effective medications for treating symptoms in all IBS subtypes include tricyclic antidepressants (TCAs) [2], antispasmodics [3], selective serotonin reuptake inhibitors (SSRIs) [4], fiber supplements, loperamide, rifaximin, pregabalin, and complementary and alternative medicine therapies such as probiotics, herbal therapies [5] and acupuncture $[6,7]$. According to evidence, until this time no complete effective curable drug is known for IBS and thus most of the drugs are still in evaluation. In recent years many drugs from synthetic or natural sources have been introduced. Most researchers in recent years have focused on phosphodiesterase inhibitors [8], melatonin [9], herbal products [5], and probiotics [10].

In the last decade, many studies have been conducted to determine the efficacy of probiotics in both experimental and clinical studies. The recommendation to use probiotics for gut disorders dates back to many years ago.

The original observation of the positive role of probiotics played by certain bacteria was first introduced in the early $20^{\text {th }}$ century by Dr Metchnikoff, who proposed the hypothesis that the aging process results from the activity of proteolytic microbes producing toxic substances in the large bowel. The proteolytic bacteria such as clostridia are part of the normal gut flora but usually produce toxic substances by digesting proteins [11]. Probiotics as live microorganisms can enhance health to the host by preventing or treating specific pathological conditions. Besides IBS, probiotics are found to be effective in decreasing duration of diarrhea and fever, especially in children [12]. Probiotics are effective in ulcerative colitis and their effectiveness in maintaining remission and preventing relapse is com-

\author{
Corresponding author: \\ Prof. Mohammad Abdollahi \\ Faculty of Pharmacy \\ and Pharmaceutical Sciences \\ Research Center \\ Tehran University \\ of Medical Sciences \\ Tehran 1417614411, Iran \\ E-mail: mohammad.abdollahi@ \\ utoronto.ca
}


parable with mesalazine [13] and only special preparations of probiotics have been suggested to be effective in Crohn's disease (CD) [14]. In agreement with this idea, the benefit of probiotics in the management of pouchitis after ileal pouch anal anastomosis (IPAA) operation was confirmed by metaanalysis $[15,16]$. It is believed that pouchitis shares many common clinical and histological features with inflammatory bowel disease (IBD) [17].

Most effective known probiotics are prepared from the genera Lactobacillus and Bifidobacterium but others such as nonpathogenic strains of Escherichia coli, Bacillus, Enterococcus, Streptococcus thermophilus, and yeasts such as Saccharomyces boulardii have evidence of effectiveness too [18]. One of the main uses of probiotics is to prepare fermented dairy products such as yogurt, freeze-dried cultures, or fermented meats and vegetables. Also capsules and tablets containing the bacteria in freeze dried form are newly available.

Depending on the species, strains, preparations, dosage and route of administration, several mechanisms have been proposed for efficacy of probiotics but in IBS they are thought to act through increasing barrier function of intestinal epithelium, inhibition of pathogenic bacteria binding to the intestinal epithelial cells, acidification of the colon and suppression of growth of the pathogens, modulation of immunity, inhibition of visceral hypersensitivity, alteration in mucosal response to stress, and improving bowel dysmotility $[18,19]$.

In recent years, many clinical trials have been conducted on efficacy of probiotics in IBS patients but most of them have been heterogenic in species, doses used, and clinical endpoints. Two broad metaanalysis studies $[10,20]$ were conducted in recent years with somewhat different concerns about homogeneity of included studies [21]. Despite the diversity in results of the studies, both meta-analyses concluded that probiotics improve symptoms of IBS and thus can be used as a supplement to standard therapy in patients with IBS. These metaanalyses showed that some strains of probiotics have some beneficial effects in patients with IBS.

Our evaluation indicates that most of the clinical trials have some defects and are not concordant in doses used, duration of treatment, inclusion criteria of patients (age, gender, race, lifestyle, and compliance), type of probiotic used, and various disease severity, leading to different results and various reports of effectiveness. But taken as a whole, probiotics are generally more effective than placebo, although in some studies the overall success rates for probiotic treatment are not high.

Of course, further research is needed to help identify the targeted patients and symptoms with use of the most effective probiotic strains and species, preferred regimen of intervention such as single or mixed species, dosage and duration of treatment. The use of probiotics for IBS warrants trials of larger size and longer duration, particularly given the chronic nature of this condition, its major impacts on patient quality of life and dearth of other effective treatments. Also researchers must deepen and widen understanding of the effects of probiotics on a basic physiological level. This therapeutic approach is undergoing constant improvement and development. Our belief is that probiotics will be ultimately a routine therapy in patients with IBS.

\section{References}

1. Khan S, Chang L. Diagnosis and management of IBS. Nat Rev Gastroenterol Hepatol 2010; 7: 565-81.

2. Rahimi R, Nikfar S, Rezaie A, Abdollahi M. Efficacy of tricyclic antidepressants in irritable bowel syndrome: a meta-analysis. World J Gastroenterol 2009; 15: 1548-53.

3. Darvish-Damavandi M, Nikfar S, Abdollahi M. A systematic review of efficacy and tolerability of mebeverine in irritable bowel syndrome. World J Gastroenterol 2010; 16: 547-53.

4. Rahimi R, Nikfar S, Abdollahi M. Selective serotonin reuptake inhibitors for the management of irritable bowel syndrome: a meta-analysis of randomized controlled trials. Arch Med Sci 2008; 4: 71-6.

5. Rahimi R, Abdollahi M. Herbal medicines for the management of irritable bowel syndrome: a comprehensive review. World J Gastroenterol 2012; 18: 589-600.

6. Trinkley KE, Nahata MC. Treatment of irritable bowel syndrome. J Clin Pharm Ther 2011; 36: 275-82.

7. Chey WD, Maneerattaporn M, Saad R. Pharmacologic and complementary and alternative medicine therapies for irritable bowel syndrome. Gut Liver 2011; 5: 253-66.

8. Salari P, Abdollahi M. Phosphodiesterase inhibitors in inflammatory bowel disease. Expert Opin Investig Drugs 2012; 21: 261-4.

9. Mozaffari S, Abdollahi M. Melatonin, a promising supplement in inflammatory bowel disease: a comprehensive review of evidences. Curr Pharm Des 2011; 17: 4372-8.

10. Nikfar S, Rahimi R, Rahimi F, Derakhshani S, Abdollahi M. Efficacy of probiotics in irritable bowel syndrome: a metaanalysis of randomized, controlled trials. Dis Colon Rectum 2008; 51: 1775-80.

11. http://en.wikipedia.org/wiki/Probiotic\#History; accessed 2/27/2012

12. Salari P, Nikfar S, Abdollahi M. A meta-analysis and systematic review on the effect of probiotics in acute diarrhea. Inflamm Allergy Drug Targets 2012; 11: 3-14.

13. Rahimi R, Nikfar S, Rezaie A, Abdollahi M. A meta-analysis of the benefit of probiotics in maintaining remission of human ulcerative colitis: evidence for prevention of disease relapse and maintenance of remission. Arch Med Sci 2008; 4: 185-90.

14. Rahimi R, Nikfar S, Rahimi F, et al. A meta-analysis on the efficacy of probiotics for maintenance of remission and prevention of clinical and endoscopic relapse in Crohn's disease. Dig Dis Sci 2008; 53: 2524-31.

15. Elahi B, Nikfar S, Derakhshani S, Vafaie M, Abdollahi M. On the benefit of probiotics in the management of pouchitis in patients underwent ileal pouch anal anastomosis: a meta-analysis of controlled clinical trials. Dig Dis Sci 2008; 53: 1278-84. 
16. Nikfar S, Darvish-Damavandi M, Abdollahi M. A review and meta-analysis of the efficacy of antibiotics and probiotics in management of pouchitis. Int J Pharmacol 2010; 6: 826-35.

17. Sandborn WJ, McLeod R, Jewell DP. Medical therapy for induction and maintenance of remission in pouchitis: a systematic review. Inflamm Bowel Dis 1999; 5: 33-9.

18. Upadhyay N, Moudgal V. Probiotics: a review. JCOM 2012; 19: 76-84.

19. Aragon G, Graham DB, Borum M, Doman DB. Probiotic therapy for irritable bowel syndrome. Gastroenterol Hepatol (NY) 2010; 6: 39-44.

20. Moayyedi P, Ford AC, Talley NJ, et al. The efficacy of probiotics in the treatment of irritable bowel syndrome: a systematic review. Gut 2010; 59: 325-32.

21. Nikfar S, Abdollahi M. The authors reply. Dis Colon Rectum 2009; 52: 1806. 